\title{
Noninvasive imaging diagnosis of sinusoidal obstruction syndrome: a pictorial review
}

\author{
Yun Zhang ${ }^{1}$, Yuling Yan $^{2}$ and Bin Song ${ }^{1 *}$ (D)
}

\begin{abstract}
Sinusoidal obstruction syndrome (SOS) is a rare liver disorder due to hepatic vascular injury. Its rapid and accurate diagnosis is crucial for patient survival. SOS is often established clinically, based on Baltimore, modified Seattle, or European Society for Blood and Marrow Transplantation (EBMT) criteria. Unfortunately, such criteria are not highly specificity and fail to provide a timely, reliable differential diagnosis. The use of noninvasive imaging techniques, such as ultrasound (US), computed tomography (CT), magnetic resonance imaging (MRI), and fluorodeoxyglucose positron emission tomography/computed tomography (FDG-PET/CT), has recently grown in this setting, some key imaging features offering diagnostic improvement. This review provides a synopsis of current noninvasive imaging techniques used for this purpose, summarizing accurate and reliable diagnostic features of SOS.
\end{abstract}

Keywords: Sinusoidal obstruction syndrome, Sinusoidal endothelial cells, Diagnostic criteria, Imaging studies, BuddChiari syndrome

\section{Key points}

- Rapid and accurate diagnosis is crucial for SOS patient's survival.

- Hepatic hemodynamic changes and parenchymal heterogeneity are the characteristic features of SOS.

- Cellular dysfunction and portal hypertension-related complications contribute to the differential diagnosis of SOS.

- Noninvasive imaging diagnosis offering diagnostic improvement of SOS.

\section{Introduction}

Sinusoidal obstruction syndrome (SOS), also referred to as veno-occlusive disease, is a rare and highly lethal condition of the liver due to vascular injury. Hematopoietic stem cell transplantation (HSCT) is most often implicated, but SOS is also associated with drug toxicity. Common agents include medicinal herbs containing pyrrolidine alkaloids (PAs), antineoplastic drugs (e.g., 6-thioguanine,

\footnotetext{
*Correspondence: songb_radiology@163.com

${ }^{1}$ Department of Radiology, Sichuan University West China Hospital, No.37,

Guoxue Alley, Chengdu 610041, Sichuan Province, China

Full list of author information is available at the end of the article
}

methotrexate, and 6-mercaptopurine), and cytotoxic chemotherapies for treating liver metastases [1-5].

SOS is typically diagnosed on a clinical basis, using Baltimore [6], modified Seattle [7, 8], or European Society for Blood and Marrow Transplantation (EBMT) criteria [9, 10]. Hepatomegaly, ascites, and bilirubin elevation are the chief diagnostic parameters, although these clinical criteria are not highly specific [5]. In addition, severe SOS may rapidly progress to multiorgan failure before a clinical diagnosis is apparent, prohibiting timely intervention and therapeutic control. Thus, more rapid and accurate diagnostic criteria are urgently needed.

Liver biopsy is the gold standard method for confirmation of SOS. However, this approach is invasive and carries a high risk of infection, rendering its routine use impractical. The recent use of noninvasive imaging techniques, including ultrasound (US), computed tomography (CT), magnetic resonance imaging (MRI), and fluorodeoxyglucose positron emission tomography-computed tomography (FDG-PET/CT), has grown in this setting given the improved diagnostics of key imaging features [11-13]. This review is focused on pertinent imaging advancements, summarizing distinctive elements that enable an accurate and reliable diagnosis of SOS. 


\section{Pathology and pathophysiology of SOS}

SOS was first reported in 1979 as an early and serious complication of HSCT [14]. It was attributed to sinusoidal endothelial cell cytotoxicity induced by serial conditioning treatments prior to HSCT [15]. Drug toxicity damage includes chemotherapy drugs for patients with liver metastases of colorectal cancer and PA-imbued medicinal herbs (such as Tu san qi) have been increasingly reported in recent decades. A central pathogenic event of SOS is toxic damage of a hepatic sinusoidal endothelial cell, marked primarily by cleavage of sinusoidal endothelial cells and substantial congestive sinusoidal dilatation in centrilobular areas and perisinusoidal fibrosis. Sinusoidal endothelial cell injury ultimately eventuates in endothelial cell exfoliation of the terminal hepatic vein, erythrocytic decomposition, and a glut of hemosiderin within the space of Disse, promoting sinusoidal blood flow impeded and leading to portal hypertension syndrome and hepatocytes dysfunction (Fig. 1).

\section{Limitations of clinical diagnostic criteria for SOS}

Baltimore [6] and modified Seattle criteria [8] are generally used to establish a clinical diagnosis of SOS. The chief clinical parameters are hyperbilirubinemia, hepatomegaly, jaundice, ascites, or unexplained weight gain. The European Society for Blood and Marrow Transplantation (EBMT) published separate criteria for adults and children, and the update is the first to acknowledge USdetermined hemodynamic change as a reliable metric of SOS. Moreover, the Chinese Society of Gastroenterology
Committee of Hepatobiliary Disease has addressed a void in terms of managing PA-related SOS by issuing the Nanjing criteria (2019) [16]. However, some instances of SOS are late in onset, and there is low disease specificity, an array of disorders presenting similarly. Thus, regardless of the improvements made and the broader applicability of established criteria, the diagnostic specificity of these criteria (rooted in clinical symptoms) is still problematic, so false-positive rates are unavoidably high.

\section{Noninvasive imaging findings of SOS}

Imaging modalities, including US, CT, MRI, and FDGPET/CT, have become the first-line methods in the diagnosis of liver disease. Some exploratory studies conducted in recent years have focused on the diagnostic potential of imaging technologies in this setting. Key imaging features of SOS have been identified that provide more timely and convincing evidence of SOS, compared with clinical parameters. Furthermore, there are state-of-the-art functional MRI technologies and quantitative imaging methods capable of predicting functional damage at a cellular level, based on intrinsic hepatic changes (Table 1).

\section{Hepatic hemodynamic changes}

As an important means of monitoring blood flow, US offers more information for early and differential diagnosis of SOS, especially in asymptomatic or late-onset cases [17]. Some findings of B-mode US, including hepatosplenomegaly, gallbladder wall thickening $>6 \mathrm{~mm}$, portal diameter $>$

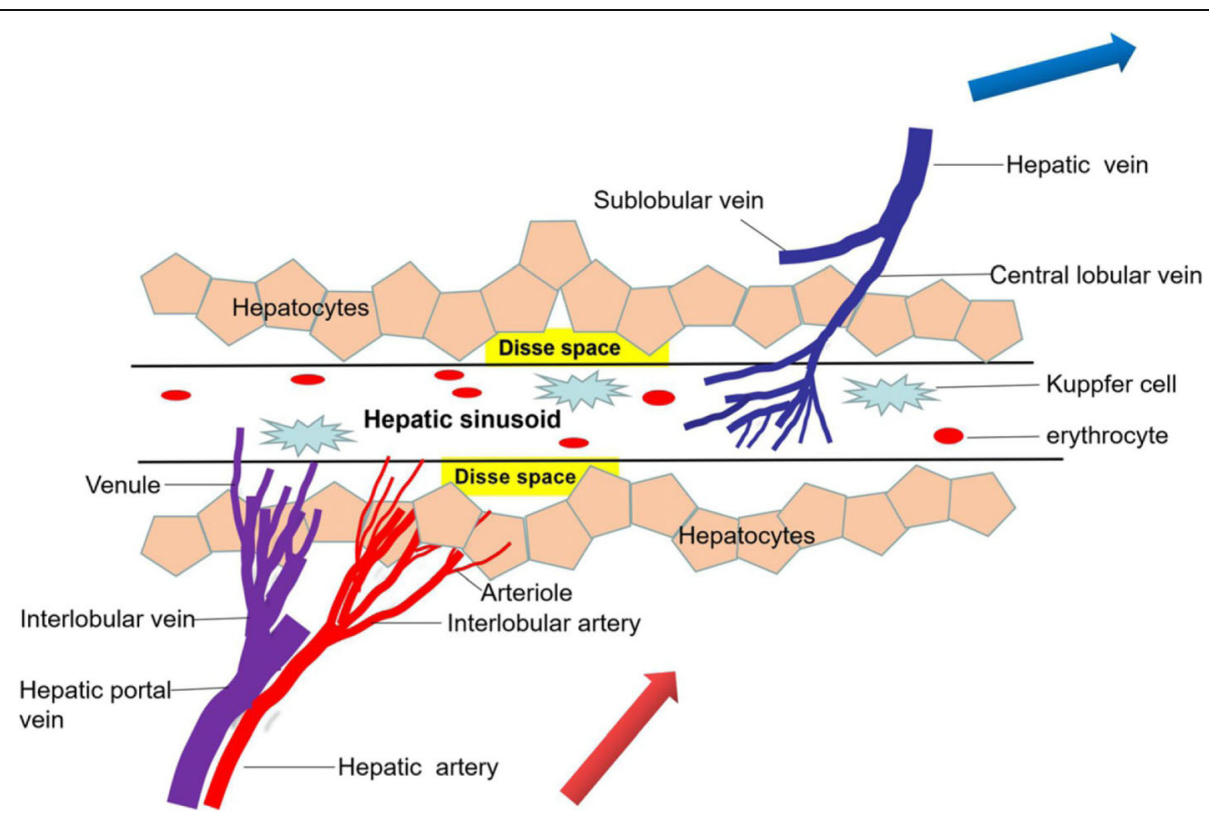

Fig. 1 Hepatic sinusoid blood flow diagram. Hepatic sinusoids are a complex of vascular conduits to be responsible for the exchange of blood, oxygen, and nutrients in the liver and systemic circulation. The structure of the hepatic sinusoids is composed of the sinusoidal endothelial cells and containing Kupffer cell. Under normal circumstances, the blood supply to the hepatic sinusoid blood come from the inlet flows (i.e., the hepatic artery and portal vein) and venous blood return to the heart through the outlet flows (i.e., the hepatic veins) 
Table 1 Different imaging modalities for SOS and their findings

\begin{tabular}{|c|c|c|c|}
\hline \multicolumn{4}{|c|}{ Major imaging modalities in diagnosis of SOS } \\
\hline \multicolumn{2}{|c|}{ Modality } & \multirow{2}{*}{$\begin{array}{l}\text { Role } \\
\text { Screening and early differential diagnosis, } \\
\text { especially in asymptomatic or late-onset } \\
\text { cases }\end{array}$} & \multirow{2}{*}{$\begin{array}{l}\text { Imaging features of SOS } \\
\text { Hepatosplenomegaly, gallbladder wall thickening }>6 \mathrm{~mm} \text {, portal diameter }> \\
12 \mathrm{~mm} \text {, hepatic vein diameter }<3 \mathrm{~mm} \text {, and indirect signs suggest portal } \\
\text { hypertension such as ascites and visualization of collateral circulatory }\end{array}$} \\
\hline US & B-mode US & & \\
\hline & Doppler US & $\begin{array}{l}\text { Surveillance and diagnostic, revealing } \\
\text { morphologic changes, and flow velocity } \\
\text { fluctuation }\end{array}$ & $\begin{array}{l}\text { Hepatic vein diameter }<3 \mathrm{~mm} \text {, collateral circulatory visualization, } \\
\text { demodulation of portal vein flow, spectral density decline, congestion } \\
\text { index }<0.1 \text {, portal vein flow }<10 \mathrm{~cm} / \mathrm{s} \text {, hepatic artery resistive index }> \\
0.75 \text {, and monophasic flow in hepatic veins }\end{array}$ \\
\hline & CEUS & Rapid diagnosis and differential diagnosis & $\begin{array}{l}\text { Diffuse or geographic enhancement of hepatic parenchyma, with a scattering } \\
\text { of hypoecho areas; hypoechoic lesion with hypervascularity in the arterial } \\
\text { phase and a rapid wash-out appearance in the portal phase }\end{array}$ \\
\hline$C T$ & & Diagnostic & $\begin{array}{l}\text { Cloverleaf or claw-like shapes; lesion with peritumoral enhancement and } \\
\text { central low attenuation }\end{array}$ \\
\hline \multirow[t]{3}{*}{ MRI } & $\begin{array}{l}\text { All gadolinium- } \\
\text { enhanced MRI }\end{array}$ & Diagnostic & $\begin{array}{l}\text { Cloverleaf or claw-like shapes; peritumoral enhancement lesion with } \\
\text { central low-signal intensity }\end{array}$ \\
\hline & $\begin{array}{l}\text { Gadoxetate- } \\
\text { enhanced MRI }\end{array}$ & $\begin{array}{l}\text { Diagnostic and provide information } \\
\text { about liver function }\end{array}$ & Diffuse or geographic hypo-intensity \\
\hline & SWI & Differential diagnosis & Geographic or nodular hypo-intensity \\
\hline
\end{tabular}

SOS sinusoidal obstruction syndrome, US ultrasound, CEUS contrast-enhanced ultrasound, CT computed tomography, MRI magnetic resonance imaging, SWI susceptibility-weighted imaging

$12 \mathrm{~mm}$, hepatic vein diameter $<3 \mathrm{~mm}$, and indirect signs suggest portal hypertension such as ascites and visualization of collateral circulatory, have been used as a routine criteria for SOS diagnosis. Doppler technique is a noninvasive method to improve the diagnostic efficiency of B-mode US by revealing morphologic changes and flow velocity fluctuation in hepatic vessels [18]. At present, the known characteristics of Doppler US of SOS include hepatic vein diameter $<3 \mathrm{~mm}$, collateral circulatory visualization, demodulation of portal vein flow, spectral density decline, congestion index $<0.1$, portal vein flow $<10 \mathrm{~cm} / \mathrm{s}$, hepatic artery resistive index $>0.75$, and monophasic flow in hepatic veins. These hemodynamic changes are closely associated with the course of disease progression. At the early stage of the disease, the sinusoidal outflow tract has a smooth blood flow allowing portal vein to maintain a normal blood flow and morphology; however, as the disease progress, hepatic venous blood flow disappears and stenosis of the main hepatic veins and portal vein blood flow is reduce and reflux occurs in some severe cases $[6,19,20]$. Dynamic monitoring of changes in the above may help predict disease progression and venture a prognosis of patients with SOS. Also, three-dimensional structures of hepatic blood vessels may be viewed using multidetector CT (MDCT)/multi-planar reformation (MPR) in combination and liver CT angiography (CTA). A disordered intrahepatic vascular network, an enlarged hepatic artery, ill-defined small arteries, and invisible hepatic veins are regular features of SOS [21, 22]. Recently, some researchers have applied FDG-PET/CT to further ascertain functional changes of the liver due to altered hepatic hemodynamics. Compared with baseline images, a rise $(\sim 10 \%)$ in standard uptake value of the liver $\left(\mathrm{SUV}_{\text {liver }}\right)$ was observed after the onset of SOS. Presumably, sinus endothelial cell injury produced altered hemodynamics and microcirculatory obstruction, disrupting inlet/outlet flow patterns in hepatic vessels. Such changes then induce hepatic congestion and passively increase hepatic blood-pool FDG tracer activity [23].

\section{Hepatic parenchymal heterogeneity}

Diffuse, patchy, or geographic heterogeneity of hepatic parenchyma, with a scattering of hypodense areas, is typical of SOS (Figs. 2, 3, and 4). Past studies indicate that parenchymal heterogeneity scores positively correlate with SOS clinical severity $[13,24,25]$. In patients with hepatic metastases of colon cancer, the efficacy of oxaliplatin-based chemotherapy is impacted by the existing SOS, and the severity of parenchymal heterogeneity is closely associated with a poor tumor response [26, 27]. Among all the heterogeneity features on enhanced CT or MRI images, cloverleaf or claw-like shapes are two classic manifestations of SOS [28]. These manifestations refer to the markedly enhanced area around the main hepatic veins which shows increased blood supply compared to the normal liver parenchyma. The appearance of characteristic shapes perhaps related to the small vascular channels surrounding the main hepatic veins. Because the initial lesion of SOS is located in hepatic sinusoid, which makes central lobular vein and sublobular vein become the first to be involved. As the disease worsens, obvious edema and necrosis of adjacent hepatocytes lead to the decrease of hepatic parenchymal enhancement; however, the small vascular channels adjacent to the main hepatic vein are kept unobstructed and 


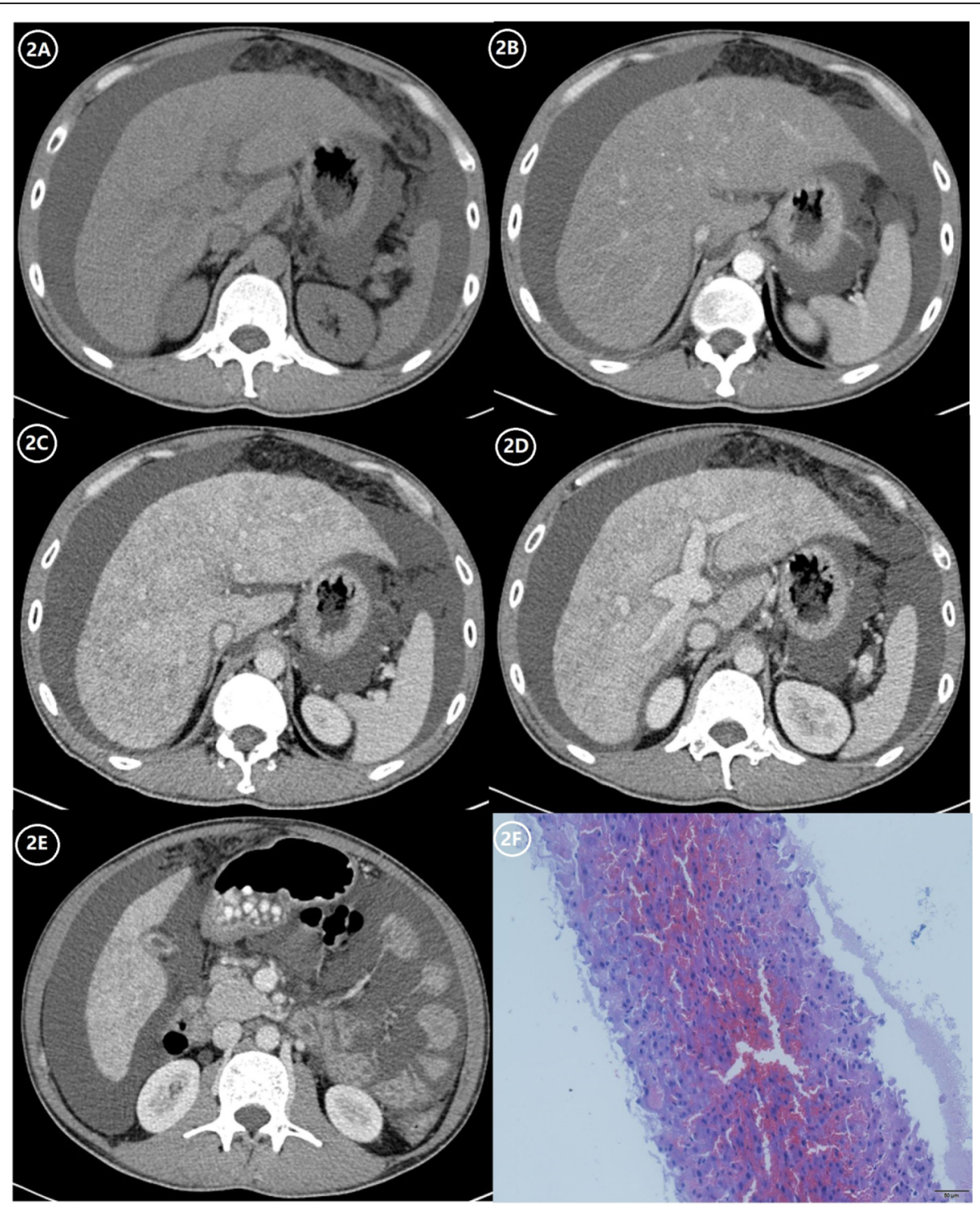

Fig. 2 A 39-year-old man with SOS caused by Tu san qi. a Normal CT scan shows non-uniform density of the liver parenchyma, hepatomegaly, and medium to a large amount of ascites. $\mathbf{b}$ Arterial phase contrast-enhanced CT scan shows non-uniform enhancement of hepatic parenchyma. c-e Portal phase contrast-enhanced CT scan shows patchy low-density lesions with a non-uniform decrease of hepatic parenchyma enhancement (the CT value of the liver is lower than that of the spleen), portal hypertension combined with collateral circulation development, gallbladder wall edema, and edema around the portal vein. $\mathbf{f}$ The hematoxylin-eosin (HE) staining at $\times 200$ magnification shows expansion and congestion around the central hepatic vein and hepatic sinus, hepatocyte edema, and mild central venous fibrosis, which is confirmed to be SOS

result in the increase of enhancement of the liver parenchyma adjacent to the venules [12]. Recently, the hepatic pseudotumor of SOS has been noted after oxaliplatin treatment $[22,29]$. Lesion of this sort is pathologically characterized by sinusoidal dilatation and congestion of hepatocytes with inflammatory cellular infiltration and areas of fibrosis [30]. On liver imaging, pseudotumor of SOS may simulate focal nodular hyperplasia, atypical HCC, or liver metastases because of the variety of enhancement patterns due to the pathological changes in the disease progression [30] (Fig. 5). On contrast- enhanced US, pseudotumor of SOS usually presents as an ill-defined hypoechoic lesion with a uniform and obvious hypervascularity in the arterial phase and a rapid wash-out appearance in the portal phase. On multiphase dynamic-enhanced CT or MRI, an irregular lesion with peritumoral enhancement and central low attenuation/signal intensity is the most common manifestation of pseudotumor of SOS. For further improving differential diagnostic efficiency between pseudotumor of SOS and malignancy, a qualitative imaging modality, such as diffuse-weighted imaging (DWI) is recommended, which 


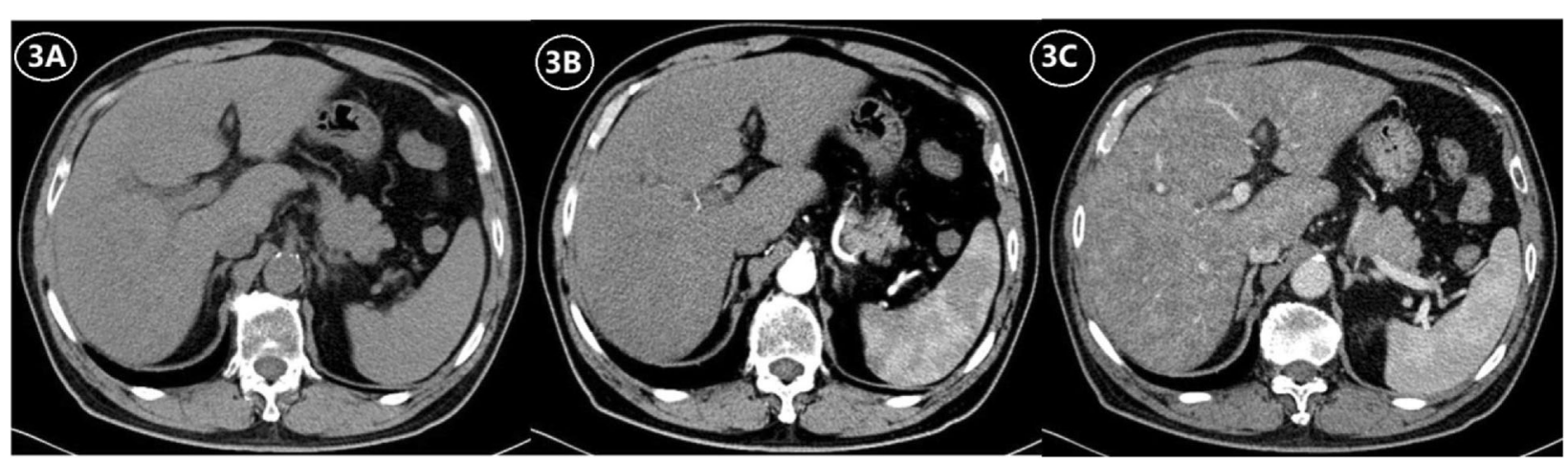

Fig. 3 A 68-year-old man with SOS caused by Tu san qi. a Normal CT scan shows a non-uniform density of the liver parenchyma and mild hepatosplenomegaly. b Arterial phase contrast-enhanced CT scan shows a small and ill-defined hepatic artery and decrease of hepatic parenchyma enhancement. c Portal phase contrast-enhanced CT scan shows patchy low-density lesions with a non-uniform decrease of hepatic parenchyma enhancement (the CT value of the liver is lower than that of the spleen)

plays a role in the theoretical basis of the cellular density difference [23].

\section{Impairment of cellular function}

Susceptibility-weighted imaging (SWI) is a quantitative imaging method that exploits differences in magnetic sensitivity to evaluate tissue densities. At present, SWI is advantageous in assessing various pathologies, particularly hemorrhages, micro-bleeds, or iron deposition in the brain [31]. The pivotal event (i.e., endothelial cell injury) in SOS ultimately eventuates in erythrocytic decomposition and a glut of hemosiderin within the space of Disse, potentially detectable by SWI. Some researchers have tested this theory by adding SWI sequences to routine MRI studies. The patchy low-signal intensities of resultant SWI images seem to approximate a portal venous-phase distribution, thus confirming these pathologic underpinnings and affording a new and noninvasive means for early diagnosis of SOS [32]. MRI studies are frequently enhanced by superparamagnetic iron-oxide nanoparticles (SPION-MRIs) or gadolinium ethoxybenzyl diethylenetriamine pentaacetic acid (GdEOB-DTPA) to assess the function of hepatocytes. SPIONMRIs provide a quantitative gauge of damage to Kupffer cells in the course of SOS, exhibiting fair agreement between severity of SOS and its corresponding histopathology [33]. The hepatobiliary phase (HBP) of a Gd-EOB-DTPAenhanced MRI is a better index of actual hepatocytic function. Previous investigations have shown that Gd-EOBDTPA-enhanced MRIs deliver high specificity and good interobserver consistency in evaluating the progression of SOS [34]. Furthermore, Yoneda et al. have reported a relation between organic anion transporting polypeptides (OATP) and functionality of hepatocytes [13]. They have documented SOS-related functional damage, showing that low-signal intensities on HBP images correlate positively with degrees of hepatocytic injury. Of note, gradual hepatic functional recovery after interruption of chemotherapy is verifiable by continuous HBP scanning.

\section{Liver fibrosis}

Fibroscan testing and acoustic radiation force impulse (ARFI) imaging, the most popular noninvasive transient elastography technologies, have been widely used to assess ongoing hepatic fibrosis and cirrhosis. Liver stiffness measurements (LSMs) determined by Fibroscan correspond with changes in the mechanical properties of the liver. Clinical increases in LSMs are seen in many conditions, including inflammation, fibrosis, congestion, cholestasis, and portal hypertension [35-37]. The injury to sinusoidal endothelial cells inherent in SOS promotes endothelial cell embolization and sinusoidal obstruction, leading to subendothelial fibrosis of sinusoids and venules, and eventual congestive fibrosis of the liver. Thus, LSM elevations may be useful preclinical and surveillance biomarkers of SOS [38]. Indeed, earlier data suggest a pre-transplant LSMs cut-point of $8 \mathrm{kPa}$ in predicting liver toxicity after HSCT, thus supporting the implementation of quantitative SOS diagnostics [39]. In addition, an animal experiment has indicated that liver shear-wave velocity (SWV), measured by ARFI imaging, bears a close relation to histologic scores of SOS, as well as degrees of hepatic lobular inflammation. Observed SWI outcomes were significantly higher in rats with SOS, as opposed to a matched control group [40]. Finally, hepatic SWV has been used in some studies (with promising results) as a quantitative biomarker to evaluate the treatment responses of patients with SOS [41].

\section{Signs associated with portal hypertension}

Hepatosplenomegaly, ascites, a thickened gallbladder wall, and periportal edema are the chief imaging features of SOS [42] (Fig. 2). Such manifestations are hallmarks of portal hypertension, stemming from obstructed intrahepatic venous drainage, and the events that follow (i.e., 


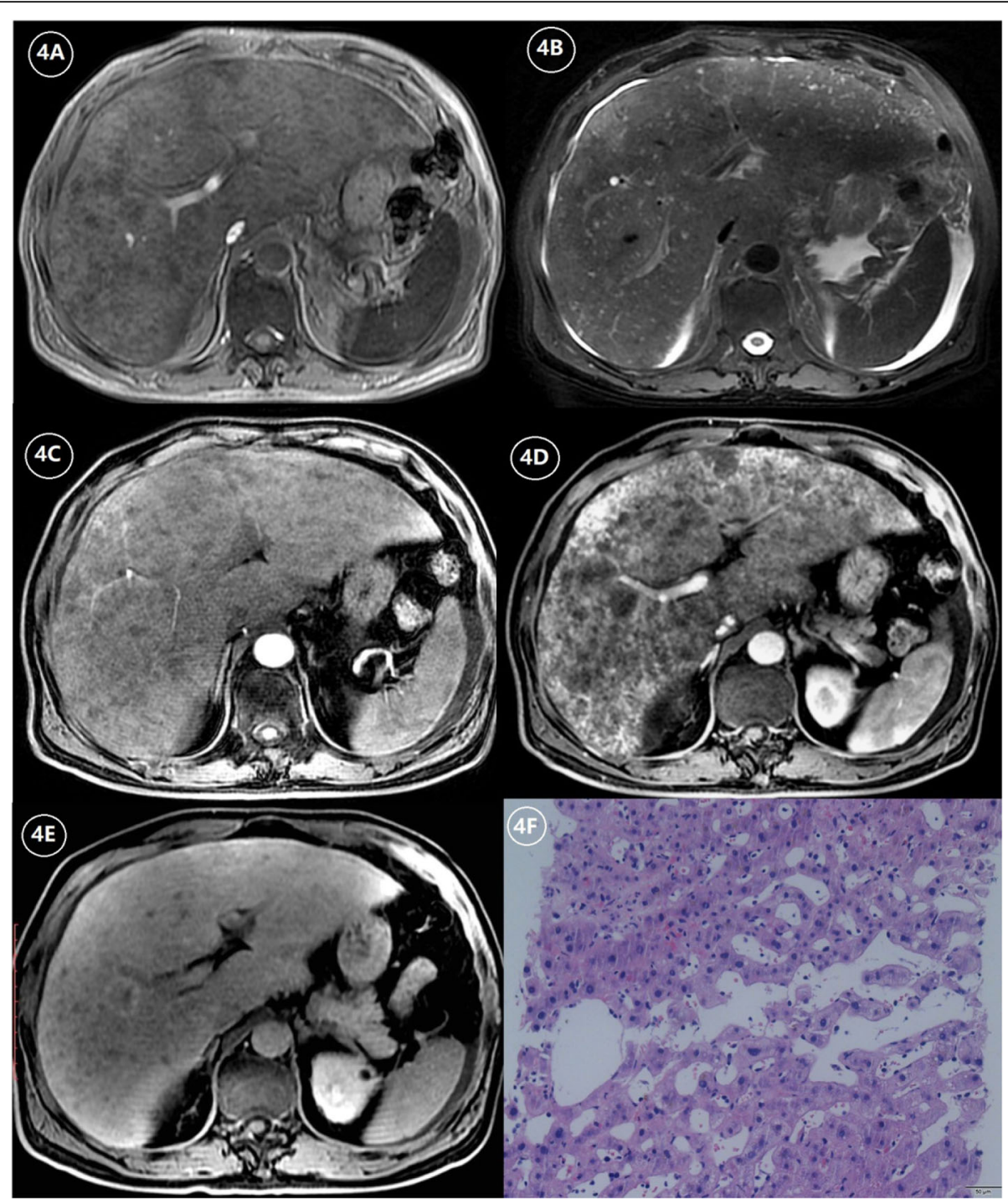

Fig. 4 a The T1-weighted image (T1WI) shows hepatomegaly, a non-uniform intensity of liver parenchyma with a patchy and nodular low-signal intensity of the liver. $\mathbf{b}$ The T2-weighted image (T2WI) shows a non-uniform signal intensity of liver parenchyma with a patchy and nodular highsignal intensity of the liver and ascites. c Arterial phase contrast-enhanced MRI scan shows heterogeneous enhancement of liver parenchyma. $\mathbf{d}$ Portal phase contrast-enhanced MRI scan shows a significant heterogeneous enhancement of liver parenchyma with patchy and nodular lesions with decreased enhancement. e HBP of gadoxetic acid-enhanced MRI shows the signal intensity of liver parenchyma is slightly decreased and accompanied by the nodular lesions with lower-signal intensity. $\mathbf{f}$ The hematoxylin-eosin (HE) staining at $\times 200$ magnification shows hepatocyte edema and significant hepatic sinus expansion, which is confirmed to be SOS

congestion of the liver and spleen, loss of liver compliance, and hepatic injury). Because many diseases are similar in terms of clinical and laboratory parameters, Erturk et al. consider narrowed hepatic veins and collateral aspects of portal hypertension to be the features distinguishing SOS from other conditions [43].

\section{Differential diagnosis of SOS}

Differential diagnosis of SOS includes graft-versus-hostdisease (GVHD) and other causes of hepatic venous outflow obstruction such as Budd-Chiari Syndrome (BCS). GVHD is the major complication of Allogeneic-HCST and is the leading cause of early mortality after transplantation [44]. The nature of GVHD is a T cell-mediated immune response. Although some clinical manifestations of GVHD are similar to that of SOS such as toxic reactions, weight gain, and deranged liver function tests; however, the distinctive features of GVHD are more often associated with inflammatory symptoms (e.g., pyrexia) and a series of skin illness (e.g., poikiloderma, nail dystrophy, and alopecia) [45]. At present, imaging studies have limitations in the diagnosis of GVHD, although some intestinal CT abnormalities have been found in acute GVHD after HCST in children [46]. BSC is an obstruction from 


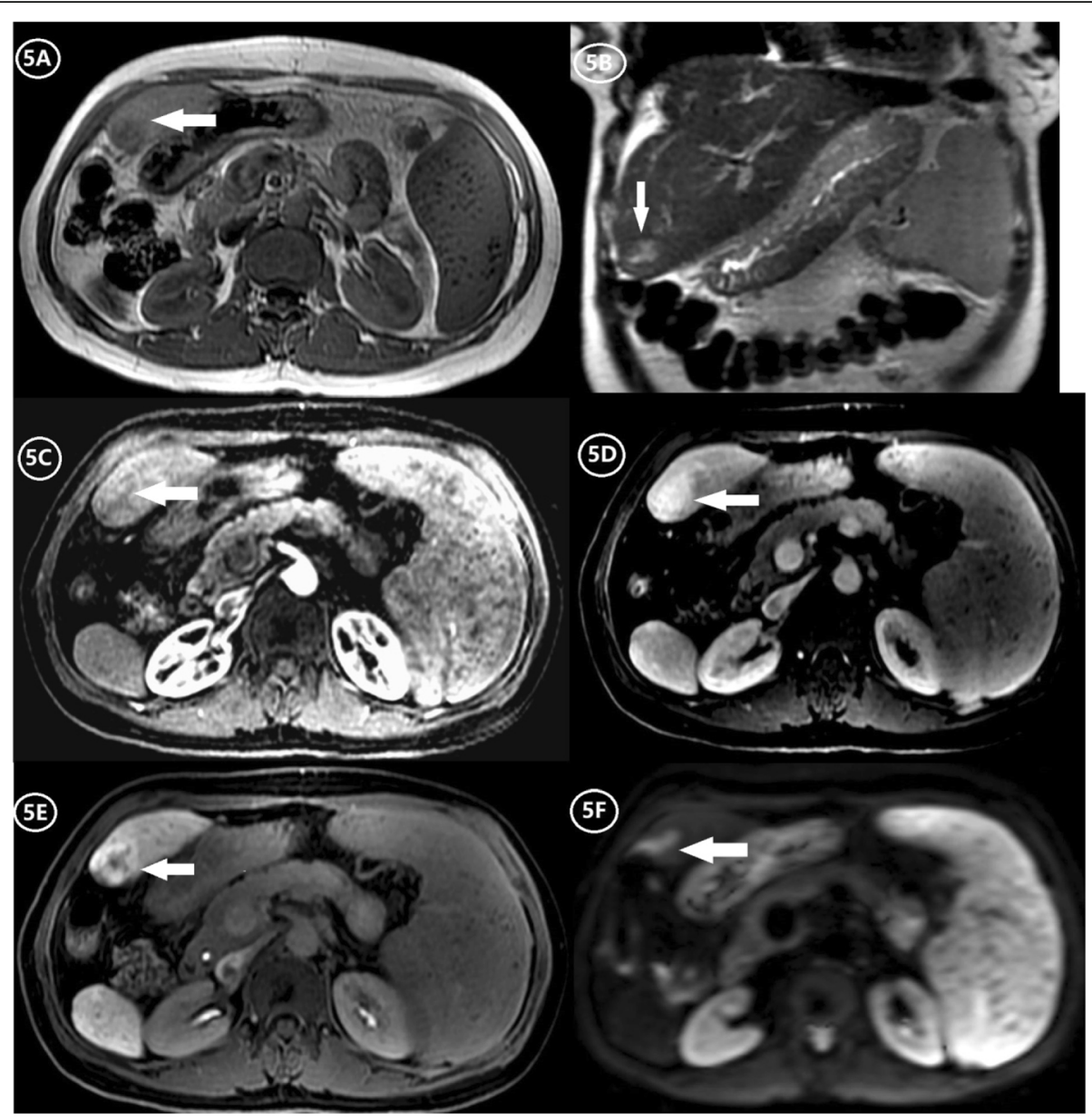

Fig. 5 A 48-year-old woman with focal SOS (arrows) developed by chemotherapy after hepatectomy. a, b A nodular lesion in liver segment III with low-signal intensity on the T1-weighted image (T1WI) and high-signal intensity on the T2-weighted image (T2WI). c Arterial phase contrastenhanced MRI scan shows a nodular lesion in liver segment III with mild peripheral enhancement. $\mathbf{d}$ Portal phase contrast-enhanced MRI scan shows the nodular lesion with apparent, uniform, and persistent enhancement. e HBP of gadoxetic acid-enhanced MRI shows the nodular lesion with a low-signal intensity at the center and high-signal intensity around it, which presents as "focal nodular hyperplasia" like lesion. $\mathbf{f}$ The diffusion-weighted imaging (DWI) shows an apparent limited diffusion

hepatic veins to the superior end of the inferior vena cava (IVC). It is characterized by centrilobular congestion and parenchymal destruction but without the endothelial cell exfoliation of the terminal hepatic vein. The primary causes of BSC include hepatic vein or IVC thrombosis, intraluminal invasion by parasite or malignancy, and extraluminal compression by solid tumor or cyst [47]. Imaging studies play an indispensable role in providing a reliable differential diagnosis between BSC and SOS. Stenosis and obstruction of hepatic veins or IVC and the presence of abnormal blood flow signals of the intrahepatic veins observed in Doppler US show good diagnostic capabilities for BSC, with a specificity of nearly $85 \%$ [48]. On contrastenhanced CT images, a "mottled appearance" along with late enhancement of the perihepatic regions of the liver and around the hepatic veins may help to differentiate SOS from BCS. This type of parenchymal heterogeneity may result from the presence of venous shunts that drain central areas and the caudate lobe of the liver. This is also the reason why an enlarged caudate lobe frequently observed in patients with BCS. MRI improves the diagnostic performance of BSC by showing collateral and a spiderweb network pattern of IVC. Moreover, early parenchymal necrosis caused by BSC and abnormal perfusion of affected liver segments could be better displayed by MRI multiple sequence scans.

\section{Conclusion}

SOS is a life-threating liver disorder related to vascular injury. At present, a timely and reliable diagnosis is unachievable using established, clinically based criteria. Recently, noninvasive imaging technologies have assumed growing importance in identifying occurrences of SOS, monitoring its progression, or predicting patient 
prognosis. Hemodynamic changes of hepatic vessels, hepatic parenchymal heterogeneity, impaired cellular function, liver fibrosis, and an array of findings signaling portal hypertension (i.e., hepatosplenomegaly, ascites, thickened gallbladder wall, and periportal edema) are all characteristic of SOS in noninvasive imaging studies. Coupled with clinical data, these imaging attributes of SOS may improve the diagnostic accuracy, especially in late-onset and asymptomatic cases, ensuring timely medical intervention.

Further prospective studies are needed to better gauge the diagnostic utility of imaging markers in terms of accuracy and specificity for SOS. Moreover, a comprehensive diagnostic and predictive model must be developed, incorporating clinical, etiologic, and imaging parameters.

\begin{abstract}
Abbreviations
ARFI: Acoustic radiation force impulse; BCS: Budd-Chiari syndrome; BCSH/ BSBMT: British Committee for Standards in Hematology/British Society for Blood and Marrow Transplantation; CT: Computed tomography; CTA: CT angiography; DWI: Diffuse-weighted imaging; EBMT: European Society for Blood and Marrow Transplantation; FDG-PET/CT: Fluorodeoxyglucose positron emission tomography-computed tomography; Gd-EOB-DTPA: Gadoliniumethoxy benzyl-diethylenetriamine pentaacetic acid; GVHD: Graft-versus-hostdisease; HBP: Hepatobiliary phase; HSCT: Hematopoietic cell transplantation; IVC: Inferior vena cava; LSMs: Liver stiffness measurements; MENA: Middle East and North Africa; MPR: Multi-planner reformation; MRI: Magnetic resonance imaging; OATP: Organic anion transporting polypeptides; PAs: Pyrrolidine alkaloids; SOS: Sinusoidal obstruction syndrome; SPIONs: Superparamagnetic iron-oxide nanoparticles; SWl: Susceptibility-weighted imaging; SWV: Shearwave velocity; US: Ultrasound
\end{abstract}

\section{Acknowledgements}

Not applicable

\section{Authors' contributions}

YZ conceptualized and designed the review together with BS. YZ and YLY drafted the initial manuscript. $Y Z$ was a major contributor in writing the manuscript. YLY proofread the pictures and table. BS reviewed and approved the final manuscript as submitted. All authors read and approved the final manuscript.

\section{Funding}

National Nature Science Foundation of China (grant number: 81771797)

\section{Availability of data and materials}

The datasets used during the current study are available from the corresponding author on a reasonable request.

\section{Ethics approval and consent to participate}

Not applicable

\section{Consent for publication}

Not applicable (the personal information in the images has been hidden)

\section{Competing interests}

The authors declare that they have no competing interests.

\section{Author details}

${ }^{1}$ Department of Radiology, Sichuan University West China Hospital, No.37, Guoxue Alley, Chengdu 610041, Sichuan Province, China. ${ }^{2}$ Department of Gastroenterology and Hepatology, Sichuan University West China Hospital, Chengdu 610041, Sichuan Province, China.
Received: 21 June 2019 Accepted: 24 September 2019

Published online: 20 November 2019

\section{References}

1. Coppell JA, Richardson PG, Soiffer R et al (2010) Hepatic veno-occlusive disease following stem cell transplantation: incidence, clinical course, and outcome. Biol Blood Marrow Transplant 16:157-168

2. Wang JY, Gao H (2014) Tusanqi and hepatic sinusoidal obstruction syndrome. J Dig Dis 15:105-107

3. Marasco G, Scaioli E, Renzulli M et al (2016) MRI Patterns in a Case of 6Thioguanine-Related Hepatic Sinusoidal Obstruction Syndrome. Am J Gastroenterol 111:767

4. McNerney KO, Vasquez JC, Kent MW, McNamara JM (2017) Sinusoidal Obstruction Syndrome During Maintenance Therapy for Acute Lymphoblastic Leukemia With 6-Mercaptopurine and Methotrexate: A Pediatric Case Report. J Pediatric Hematol Oncol 39:e454-e455

5. Robinson SM, Wilson CH, Burt AD, Manas DM, White SA (2012) Chemotherapy-associated liver injury in patients with colorectal liver metastases: a systematic review and meta-analysis. Ann Surg Oncol 19: 4287-4299

6. Jones RJ, Lee KS, Beschorner WE et al (1987) Venoocclusive disease of the liver following bone marrow transplantation. Transplantation 44:778-783

7. Shulman HM, Hinterberger W (1992) Hepatic veno-occlusive disease--liver toxicity syndrome after bone marrow transplantation. Bone Marrow Transplant 10:197-214

8. McDonald GB, Hinds MS, Fisher LD et al (1993) Veno-occlusive disease of the liver and multiorgan failure after bone marrow transplantation: a cohort study of 355 patients. Ann Intern Med 118:255-267

9. Mohty M, Malard F, Abecassis M et al (2016) Revised diagnosis and severity criteria for sinusoidal obstruction syndrome/veno-occlusive disease in adult patients: a new classification from the European Society for Blood and Marrow Transplantation. Bone Marrow Transplant 51:906-912

10. Corbacioglu S, Carreras E, Ansari M et al (2018) Diagnosis and severity criteria for sinusoidal obstruction syndrome/veno-occlusive disease in pediatric patients: a new classification from the European society for blood and marrow transplantation. Bone Marrow Transplant 53:138-145

11. Abadia Barno M, Marin Serrano E, Ruiz Fernandez G (2017) Use of abdominal ultrasound in the diagnosis of hepatic sinusoidal obstruction syndrome (SOS) in a patient receiving a hematopoietic-cell transplant. Rev Esp Enferm Dig 109:608-609

12. Zhou H, Wang YX, Lou HY, Xu XJ, Zhang MM (2014) Hepatic sinusoidal obstruction syndrome caused by herbal medicine: $C T$ and MRI features. Korean J Radiol 15:218-225

13. Yoneda N, Matsui O, Ikeno H et al (2015) Correlation between Gd-EOBDTPA-enhanced MR imaging findings and OATP1B3 expression in chemotherapy-associated sinusoidal obstruction syndrome. Abdominal Imaging 40:3099-3103

14. Jacobs P, Miller JL, Uys CJ, Dietrich BE (1979) Fatal veno-occlusive disease of the liver after chemotherapy, whole-body irradiation and bone marrow transplantation for refractory acute leukaemia. S Afr Med J 55:5-10

15. Tuncer HH, Rana N, Milani C, Darko A, Al-Homsi SA (2012) Gastrointestinal and hepatic complications of hematopoietic stem cell transplantation. World J Gastroenterol 18:1851-1860

16. Zhuge Y, Liu Y, Xie W, Zou X, Xu J, Wang J (2019) Expert consensus on the clinical management of pyrrolizidine alkaloid-induced hepatic sinusoidal obstruction syndrome. J Gastroenterol Hepatol 34:634-642

17. Dietrich CF, Trenker C, Fontanilla T et al (2018) New Ultrasound Techniques Challenge the Diagnosis of Sinusoidal Obstruction Syndrome. Ultrasound Med Biol 44:2171-2182

18. Corvino A, Sandomenico F, Corvino F et al (2019) Utility of a gel stand-off pad in the detection of Doppler signal on focal nodular lesions of the skin. J Ultrasound. https://doi.org/10.1007/s40477-019-00376-3 [PMID: 30927249 Epub ahead of print]

19. Lassau N, Leclere J, Auperin A et al (1997) Hepatic veno-occlusive disease after myeloablative treatment and bone marrow transplantation: Value of gray-scale and Doppler US in 100 patients. Radiology 204:545-552

20. Herbetko J, Grigg AP, Buckley AR, Phillips GL (1992) Venoocclusive liver disease after bone marrow transplantation: findings at duplex sonography. AJR Am J Roentgenol 158:1001-1005 
21. Uchino K, Fujisawa M, Watanabe T et al (2013) Oxaliplatin-induced liver injury mimicking metastatic tumor on images: a case report. Jpn J Clin Oncol 43:1034-1038

22. Kawai T, Yamazaki S, Iwama A, Higaki T, Sugitani M, Takayama T (2016) Focal Sinusoidal Obstruction Syndrome Caused by Oxaliplatin-Induced Chemotherapy: A Case Report. Hepat Mon 16:e37572

23. Kim H, Baek SE, Moon J, Roh YH, Lee N, Cho A (2016) Increased hepatic FDG uptake on PET/CT in hepatic sinusoidal obstructive syndrome. Oncotarget 7:69024-69031

24. Arakawa Y, Shimada M, Utsunomya T et al (2013) Oxaliplatin-related sinusoidal obstruction syndrome mimicking metastatic liver tumors. Hepatol Res 43:685-689

25. Shao H, Chen HZ, Zhu JS et al (2015) Computed tomography findings of hepatic veno-occlusive disease caused by Sedum aizoon with histopathological correlation. Braz J Med Biol Res 48:1145-1150

26. Vigano L, Capussotti L, De Rosa G, De Saussure WO, Mentha G, RubbiaBrandt L (2013) Liver resection for colorectal metastases after chemotherapy: impact of chemotherapy-related liver injuries, pathological tumor response, and micrometastases on long-term survival. Ann Surg 258: 731-740 discussion 741-732

27. Han NY, Park BJ, Kim MJ, Sung DJ, Cho SB (2015) Hepatic Parenchymal Heterogeneity on Contrast-enhanced CT Scans Following Oxaliplatin-based Chemotherapy: Natural History and Association with Clinical Evidence of Sinusoidal Obstruction Syndrome. Radiology 276:766-774

28. Cayet S, Pasco J, Dujardin F et al (2017) Diagnostic performance of contrastenhanced CT-scan in sinusoidal obstruction syndrome induced by chemotherapy of colorectal liver metastases: Radio-pathological correlation. Eur J Radiol 94:180-190

29. Elsayes KM, Menias CO, Morshid Al et al (2018) Spectrum of Pitfalls, Pseudolesions, and Misdiagnoses in Noncirrhotic Liver. AJR Am J Roentgenol 211:97-108

30. Guarino B, Catalano O, Corvino A, Corvino F, Amore A, Petrillo A (2015) Hepatic inflammatory pseudotumor: educational value of an incorrect diagnosis at contrast-enhanced ultrasound. J Med Ultrason (2001) 42:547552

31. Liu S, Buch S, Chen Y et al (2017) Susceptibility-weighted imaging: current status and future directions. NMR Biomed 30:e3552

32. Guo T, Li X, Yang X, et al (2019) Gadoxetic Acid-Enhanced HepatobiliaryPhase Magnetic Resonance Imaging for Pyrrolizidine Alkaloid-Induced Hepatic Sinusoidal Obstruction Syndrome and Association with Liver Function. Sci Rep 9:1231

33. Ward J, Guthrie JA, Sheridan MB et al (2008) Sinusoidal obstructive syndrome diagnosed with superparamagnetic iron oxide-enhanced magnetic resonance imaging in patients with chemotherapy-treated colorectal liver metastases. J Clin Oncol 26:4304-4310

34. Shin NY, Kim MJ, Lim JS et al (2012) Accuracy of gadoxetic acid-enhanced magnetic resonance imaging for the diagnosis of sinusoidal obstruction syndrome in patients with chemotherapy-treated colorectal liver metastases. Eur Radiol 22:864-871

35. Coco B, Oliveri F, Maina AM et al (2007) Transient elastography: a new surrogate marker of liver fibrosis influenced by major changes of transaminases. J Viral Hepat 14:360-369

36. Millonig G, Friedrich S, Adolf S et al (2010) Liver stiffness is directly influenced by central venous pressure. J Hepatol 52:206-210

37. Millonig G, Reimann FM, Friedrich S et al (2008) Extrahepatic cholestasis increases liver stiffness (FibroScan) irrespective of fibrosis. Hepatology 48 1718-1723

38. Colecchia A, Ravaioli F, Sessa MD et al (2019) Liver Stiffness Measurement Allows Early Diagnosis of Veno-Occlusive Disease/Sinusoidal Obstruction Syndrome in Adult Patients Who Undergo Hematopoietic Stem Cell Transplantation: Results from a Monocentric Prospective Study. Biol Blood Marrow Transplant 25:995-1003

39. Auberger J, Graziadei I, Clausen J, Vogel W, Nachbaur D (2013) Non-invasive transient elastography for the prediction of liver toxicity following hematopoietic SCT. Bone Marrow Transplant 48:159-160

40. Park SH, Lee SS, Sung JY et al (2018) Noninvasive assessment of hepatic sinusoidal obstructive syndrome using acoustic radiation force impulse elastography imaging: A proof-of-concept study in rat models. Eur Radiol 28:2096-2106

41. Fontanilla T, Hernando CG, Claros JC et al (2011) Acoustic radiation force impulse elastography and contrast-enhanced sonography of sinusoidal obstructive syndrome (Veno-occlusive Disease): preliminary results. Ultrasound Med 30:1593-1598

42. Mahgerefteh SY, Sosna J, Bogot N, Shapira MY, Pappo O, Bloom Al (2011) Radiologic imaging and intervention for gastrointestinal and hepatic complications of hematopoietic stem cell transplantation. Radiology 258: 660-671

43. Erturk SM, Mortele KJ, Binkert CA et al (2006) CT features of hepatic venoocclusive disease and hepatic graft-versus-host disease in patients after hematopoietic stem cell transplantation. AJR Am J Roentgenol 186:1497-1501

44. Mannina D, Kroger N (2019) Janus Kinase Inhibition for Graft-Versus-Host Disease: Current Status and Future Prospects. Drugs 79(14):1499-1509

45. Dignan FL, Fau AP, Clark A et al (2012) Diagnosis and management of chronic graft-versus-host disease. Br J Haematol 158:46-61

46. Arpaci $\mathrm{T}$ (2018) Computed tomography imaging of acute gastrointestinal graft-versus-host disease after haematopoietic stem cell transplantation in children. Contemp Oncol (Pozn) 22:178-183

47. Bayraktar UD, Seren S, Bayraktar Y (2007) Hepatic venous outflow obstruction: three similar syndromes. World J Gastroenterol 13:1912-1927

48. Bolondi L, Fau GS, Li Bassi S et al (1991) Diagnosis of Budd-Chiari syndrome by pulsed Doppler ultrasound. Gastroenterology 100:1324-1331

\section{Publisher's Note}

Springer Nature remains neutral with regard to jurisdictional claims in published maps and institutional affiliations.

\section{Submit your manuscript to a SpringerOpen ${ }^{\circ}$ journal and benefit from:}

- Convenient online submission

- Rigorous peer review

- Open access: articles freely available online

- High visibility within the field

- Retaining the copyright to your article

Submit your next manuscript at $>$ springeropen.com 